\title{
Signals Involved in Neural Differentiation of Human Embryonic Stem Cells
}

\author{
Mark Denham ${ }^{\mathrm{a}}$ Mirella Dottori ${ }^{\mathrm{a}, \mathrm{b}}$ \\ ${ }^{a}$ Centre for Neuroscience, and bepartment of Pharmacology, The University of Melbourne, Parkville, Vic., Australia
}

\section{Key Words}

Human embryonic stem cells • Differentiation, neural • Induction, neural $\cdot$ Maintenance, neural $\cdot$ Specification, neural $\cdot$ Dopamine neurons $\cdot$ Motor neurons

\begin{abstract}
Neural differentiation from embryonic stem cells involves progressive stages of neural induction, expansion and maintenance of neural stem/progenitor cells, and differentiation to neurons and glia. Our understanding of the signals involved in each of these processes is primarily based on our knowledge of neural development during embryogenesis. This review will focus on the signalling pathways that have been identified to play a role in neural differentiation of human embryonic stem cells ( $\mathrm{hESC}$ ), including their induction to neuroectoderm, maintenance and expansion of hESCderived neurospheres, differentiation to neurons and specification to specific neuronal lineages. Understanding the signals involved in each of these stages is important for optimising methods to derive specific cell types for transplantation therapies, as well as for providing insight into the mechanisms of human neurogenesis.
\end{abstract}

Copyright $\odot 2009$ S. Karger AG, Basel

\section{Introduction}

When human embryonic stem cell (hESC) lines were first derived in 1998 by Thomson et al. [1], there was much excitement within the scientific and public com- munity as well as ethical debates. hESC lines provide an unlimited source of human cells that can be differentiated to all cell types and at all stages of development. This is ideal for developing cell replacement therapies to treat degenerative diseases. However, this pluripotent characteristic of hESCs is a 'double-edged sword' and one of the major challenges in hESC research lies in determining how to direct hESCs to a specific progenitor cell type. For this, we need to thoroughly understand the signalling pathways involved in hESC differentiation. This review will focus on three main stages of hESC neural differentiation: neural induction, maintenance and expansion of neural progenitors, and their differentiation and specification to neurons. The signals involved in each of these stages are yet to be fully identified in hESCs and much of what is known has been based on studies of early neural development and mouse ESCs. Unravelling these pathways will help establish optimal protocols to direct hESC fate and thus provide pure populations of cell types that can be used for further research.

\section{Signals Involved in Neural Induction}

To date, there are three major methods of promoting hESC neural induction. The earliest method of generating neural stem cells (NSCs) was by keeping hESC cultures beyond a week without passage to allow their spontaneous differentiation to early progenitors of germ lineages [2]. Cells that had differentiated to neuroectoderm could be morphologically identified by their aggregation

\section{KARGER \\ Fax +41613061234 E-Mail karger@karger.ch} www.karger.com
(C) 2009 S. Karger AG, Basel

$1424-862 X / 09 / 0174-0234 \$ 26.00 / 0$

Accessible online at:

www.karger.com/nsg
Mirella Dottori, $\mathrm{PhD}$

Stem Cell Laboratory, Centre for Neuroscience and Department of Pharmacology

Level 7, West Wing, Medical Building, The University of Melbourne

Parkville, Vic. 3010 (Australia)

Tel. +61 38344 3988, Fax +61 39349 4432, E-Mail mdottori@unimelb.edu.au 
into rosette-like structures, resembling neuroepithelial cells of the embryonic neural tube. A variation to this approach is to culture hESCs in suspension such that they form aggregates of differentiated cells, known as embryoid bodies [3-6]. The media used in spontaneously differentiating cultures may also be suited for maintenance of NSCs, thus allowing a biased survival of neural progenitors in particular. Although the media may be defined in these differentiation systems, the critical factors and signalling pathways that influence neural induction in hESCs are not known. These methods may rely on the notion that hESC neurogenesis is occurring by a default endogenous pathway and the culture conditions are optimized to specifically sustain the growth and maintenance of neural cells. The second most common approach to promote hESC neural induction is to co-culture hESCs on a feeder layer of stromal cells, such as PA6 or MS5 cell lines $[7,8]$. Such stromal cell lines appear to secrete, or at least express, factors that promote hESC neural rosette formation. In these feeder layer formats it would be difficult to completely define the factors responsible for hESC neural induction.

To date, the only approach that has been shown to induce hESC neural differentiation in a defined manner is by directly inhibiting the BMP and/or SMAD signalling pathways [9]. If hESCs are left to spontaneously differentiate in vitro, one of the first lineages to arise is primitive endodermal cells. It was found that this differentiation was mediated by a positive feedback loop whereby BMP2 secreted by hESCs activated the Smad1 pathway, thereby generating their differentiation [10]. In order to inhibit this pathway and perhaps retain hESCs undifferentiated, Pera et al. [10] tested the effects of treating hESCs with different BMP antagonists including follistatin, cerberus and noggin. Only noggin treatment was shown to have any obvious effects on hESCs, although not by preventing their differentiation. Treatment of hESCs with noggin generated a homogenous, morphologically distinct population of cells and that expressed neuroectodermal markers, including Pax6 and Sox2, and no detection of mesoderm or endoderm lineage markers. Interestingly, noggin-treated hESC colonies do not show the characteristic rosette-like structures as observed in spontaneously differentiated cultures (fig. 1). However, classic neural rosettes are observed in neurospheres derived from noggintreated hESC colonies (fig. 1d, e) $[10,11]$. It may be that the noggin-treated hESCs are at an earlier stage of neurogenesis, perhaps representing a population of early neuroectodermal cells, as compared to neurospheres that may be more representative of neuroepithelial cells of the neural tube. These experiments were performed in culture conditions that required feeder layers of mouse embryonic fibroblasts and in the presence of serum media, suggesting that perhaps noggin is synergising with other undefined factors to mediate hESC neural induction $[10$, 12]. Another study showed that chemically defined media supplemented with noggin resulted in Pax6+/Sox1neural rosettes, and additional supplementation of fibroblast growth factor (FGF) induced Pax6+/Sox $1+$ neural rosettes that could differentiate into neurons [13]. This was supported by similar studies showing formation of neurospheres when hESCs were cultured in suspension in neurosphere maintenance media supplemented with noggin [14]. Thus, noggin alone appears to at least initiate hESC differentiation towards neural.

BMPs form part of the transforming growth factor $\beta$ (TGF $\beta$ ) superfamily of growth factors that signal through specific heteromeric complexes of type I and type II receptors $[15,16]$. Activation of type I and type II receptors results in phosphorylation of receptor-regulated Smad proteins. The BMP pathway mediates its signal via phosphorylation of Smad1, Smad5 and Smad8. Other TGF $\beta$ members, activin and TGF $\beta$, mediate their signal via Smad 2 and Smad3. Phosphorylated Smad proteins form a complex with Smad4 to then regulate transcription of downstream target genes. As explained above, noggin treatment in hESCs blocks the BMP/Smad1-signalling pathway. A recent study showed that hESC neural induction occurred with higher efficiency by combined treatment of noggin and the small molecule, SB431542 [9]. SB431542 inhibits phosphorylation of activin and TGF $\beta$ receptors, ALK4, ALK5 and ALK7, thereby blocking the downstream Smad2/3-signalling pathway. Thus, the combined inhibition of BMP-mediated and activin/ TGF $\beta$-mediated Smad-signalling pathway augments hESC neural induction. The next step is to determine which pathways are active to mediate transcription of early neural genes in hESCs and what other factors may be needed to induce these signalling pathways.

A commonly used method for neural induction of mouse ESCs is to treat embryoid bodies with retinoic acid (RA) [17-19]. However for hESCs, RA treatment appears to be involved in directing neural progenitor fate towards spinal cord progenitors rather than in neural induction itself [20] (see below). Thus, there may be some fundamental differences in neural induction signals between hESCs and mouse ESCs. This is consistent with the difference between hESCs and mouse ESCs in requiring LIF signalling to maintain ESCs pluripotent [21]. Saying this, other methods used for hESC neural induction are also 
applicable for mouse ESCs, including PA6 stromal coculture systems and noggin signalling [22-24]. Thus, although many signals and mechanisms involved in neural induction and differentiation may be highly conserved processes across species, one cannot presume that they are necessarily consistent. For this reason, studies in hESC neural differentiation need to be thoroughly characterized, similar to what has been previously done for mouse ESC neural differentiation.

\section{Signals Involved in Neural Expansion and Maintenance}

Once hESCs have undergone neural induction, the next stage is to maintain and expand the neural stem/ progenitor cells in culture. Following neural induction, cells are usually transferred to suspension culture conditions that promote formation of neurospheres, as adapted from the mouse neurosphere assay $[25,26]$. The media used for neurosphere formation and maintenance are commonly supplemented with mitogenic factors, bFGF and epidermal growth factor (EGF) [12].

hESC colonies that are treated with noggin for 14 days for neural induction are then mechanically isolated and cultured in suspension to form neurosphere aggregates $[10,12]$. Expression analyses of neurospheres derived from noggin-treated hESCs show different transcriptional profiles compared to noggin-treated hESC colonies. At the neurospheres stage, there is an upregulation of the dorsal neural markers, Pax 3 and Pax7, and these Pax3+1 Pax7+ cells appear to be mutually exclusive to Pax6+ cells [11]. Thus, neurospheres consist of a heterogeneous population of early neural progenitors. It was also found that at the neurosphere stage of hESC neural differentiation, there is endogenous production of canonical and non-canonical Wnt proteins as well as upregulation of Wnt receptors, suggesting a requirement of Wnt signalling for maintenance of hESC-derived neural progenitors [11]. In support of this hypothesis, exogenous treatment of Wnt3a to hESC-derived neurospheres resulted in increased efficiency of neurosphere formation and neurosphere size [11]. Likewise, inhibition of canonical Wnt signalling in neurospheres by Dkk treatment resulted in poor neurosphere formation. Activation of Wnt signalling was correlated with an increase in proliferation and survival of neural progenitors [11]. These findings suggest an autocrine mechanism of Wnt signalling by hESCderived neural progenitors to enhance their survival and proliferation. Since neurospheres consist of heteroge- neous neural progenitor subtypes and are also intrinsically differentiating within the neurospheres over time, it remains to be determined if Wnt signalling is occurring in a subset of progenitors and/or at a specific stage of the neural differentiation pathway.

Studies by Elkabetz et al. [27] demonstrated that the rosette-forming NSCs (R-NSCs) that express anterior markers of the nervous system, such as Forsel, have the broadest differentiation potential. R-NSCs were able to differentiate to cell types of both anterior-posterior, central and peripheral nervous system. Interestingly, R-NSCs that expressed caudal markers of the nervous system were more restricted in their potential to differentiate to neural cell types. Consistent results were observed with R-NSCs derived from using neural induction methods of either stromal co-cultures or by embryoid body formation [27]. The authors demonstrated that R-NSCs could be maintained long-term by stimulating the sonic hedgehog (Shh) and notch pathways; however, this maintenance was only observed in high-density cultures. Interestingly, bFGF and EGF promoted differentiation of RNSCs. This may explain why hESC-derived neurospheres maintained in media supplemented with bFGF and EGF, as described above, may consist of heterogeneous cell types. Thus, to elucidate key signalling pathways involved in maintaining hESC-derived neural stem/progenitor cells, it is desirable to first establish markers to identify progenitor subtypes in culture, and second to optimize the culture conditions such that pure populations of progenitor subtypes can be maintained. As the technology advances for hESC research, there will be more refined assay and culture systems available to address these needs.

Fig. 1. a Bright-field image of a hESC colony cultured on mouse fibroblasts and treated with noggin for 14 days for neural induction. At this stage, although neural rosettes are not observed within the colony, many of the cells within the colony express Pax6 (b). c Dapi image of $\mathbf{b}$. d Neurosphere derived from noggin-treated hESC, stained with the nuclear marker Dapi, showing rosettes (arrows). e Bright field showing hESC-derived neurosphere plated on fibronectin substrate. Rosettes are observed within the central regions (arrow). a, e Scale bar $=500 \mu \mathrm{m}$; c, $\mathbf{d}$ scale bar $=200$ $\mu \mathrm{m}$.

Fig. 2. Summary outlining the various stages of hESC neural differentiation, from neural induction of pluripotent stem cells to expansion and early specification of neural progenitors and finally their differentiation to specific neuronal lineages. 

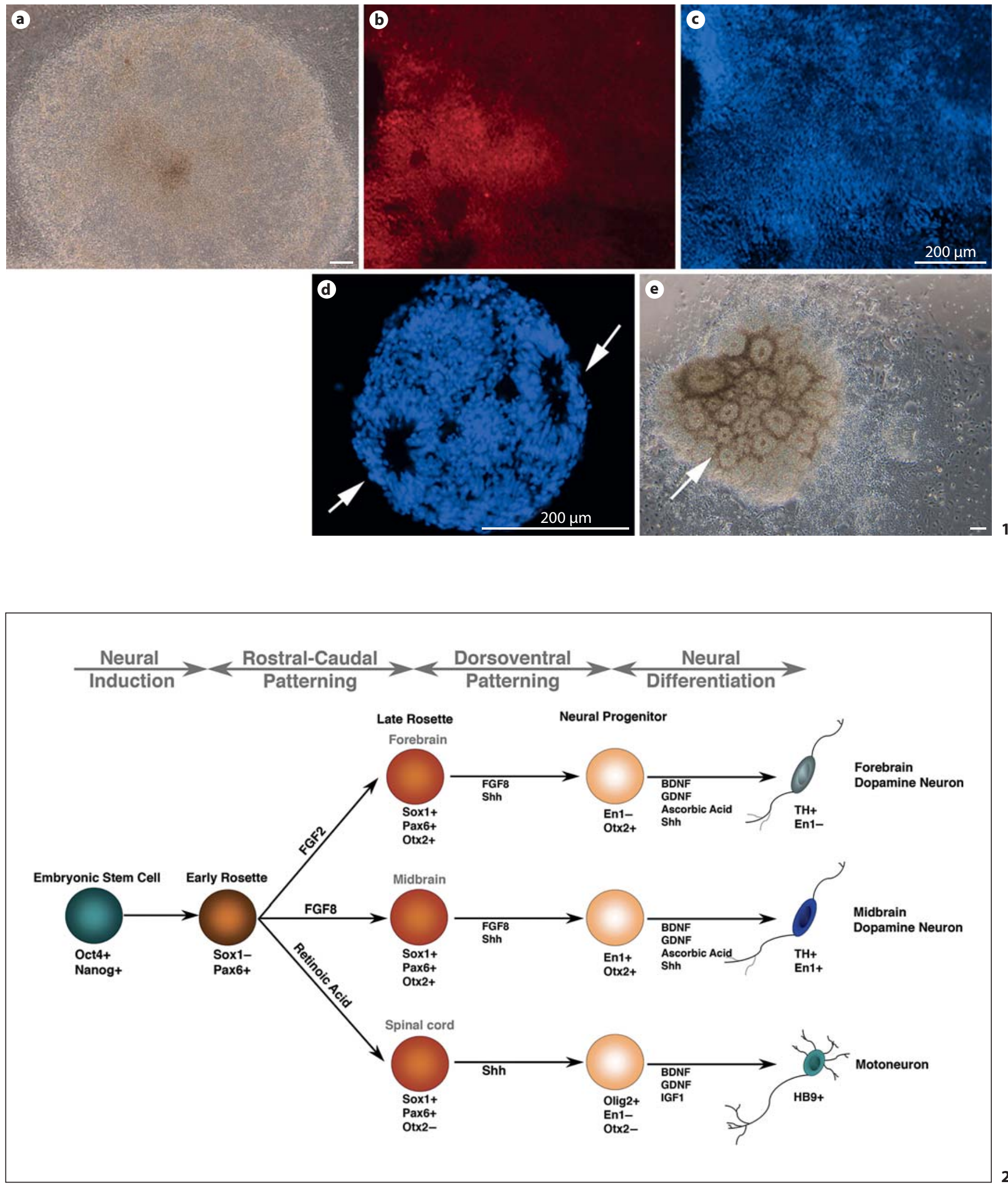

Signals Involved in Neural Differentation 


\section{Signals Involved in Neural Differentiation}

The third stage of hESC neurogenesis is differentiation of neural progenitors to neurons and glia. One of the most challenging aspects of stem cell therapies is that in vivo differentiation of NSCs tends to be biased towards glial lineages, particularly during degeneration/injury when there is extensive and ongoing inflammation. Thus, identifying the signals that can regulate neuronal differentiation may be very useful therapeutically for developing stem cell therapies to treat neurodegenerative disorders.

In vitro differentiation of hESC-derived neural progenitors usually involves plating neurospheres onto laminin and fibronectin substrates that bias their differentiation towards neural and glia, respectively [12]. Removal of mitogenic factors, bFGF and EGF, also promotes differentiation of progenitors to $\beta$-tubulin-positive neurons. Whereas for glial differentiation, neural basal media continue to be supplemented with bFGF and EGF, as well as platelet-derived growth factor-AB (PDGF-AB) [12]. The presence or absence of mitogenic factors for glial or neural differentiation, respectively, suggest that pathways involved in lineage specification may also be associated with pathways that regulate cell cycle.

Factors that are released in neuroinflammation may very likely influence the differentiation fate of NSCs. This was found to be the case with lysophospholipids. Lysophosphatidic acid (LPA) is a bioactive lipid that is released by activated platelets and is a significant factor contributing to an inflammatory response during neurotrauma [28]. Previous studies investigating the effects of lysophospholipids in hESC neural differentiation identified that LPA was able to inhibit differentiation of hESC-derived neural progenitors to neurons [29]. Interestingly, differentiation to astrocytes was not affected by LPA treatment. It was found that LPA-induced inhibition of neuronal differentiation was mediated by combined activation of the Rho/ROCK and P13K/Akt pathways, and these two pathways acted independently [29]. Thus, blocking these pathways may be a useful mechanism for inducing neuronal differentiation in hESC-derived progenitors and may be a strategy to consider in stem cell transplantation studies.

\section{Signals Involved in Neural Specification}

If stem cells are to be used in cell replacement therapies, it would be preferable to transplant a specific cell type that would functionally replace degenerated endog- enous cells. For this reason it is important to know how to direct hESC neural differentiation towards a specific lineage of the nervous system. hESC neuronal differentiation can be biased towards specific neuronal lineages by mimicking precise patterning signals during embryonic development. Patterning signals may act as agonists or antagonists to drive or block cell fate, respectively. Similar to patterning within the embryonic neural tube, neuronal specification is dependent on the concentration of the morphogen. Finally, the precise timing of exogenous signalling is critical for neuronal specification. All of these aspects need to be taken into consideration, and carefully tested, when developing a system to derive specific neuronal populations from hESCs.

\section{Dopamine Neurons}

Derivation of dopamine neurons from hESCs has been one of the primary cell types pursed as a target for developing stem cell therapies to treat Parkinson's disease [30]. Parkinson's disease is a neurodegenerative disorder that involves specific degeneration of dopamine neurons within the substantia nigra regions of the brain. During embryogenesis, dopamine neurons of the substantia nigra arise from the developing ventral midbrain, also known as the ventral mesencephalon, and can be identified by the combination of transcription factors some of which are: Pitx3, Lmx1a, Nurr1, En1, Foxa2 [31-36]. Dopamine neurons also arise in the developing forebrain regions; however, these neurons show a different transcription expression profile during development [37]. It is not yet established whether forebrain dopamine neurons are suitable for replacing function of degenerate midbrain dopamine neurons. Nevertheless, the strategy used to derive dopamine neurons from embryonic and/or NSCs is focused on generating ventral midbrain neural progenitors with the intention of enriching midbrain dopamine neurons.

Shh and FGF8 are regarded as the two key ligands involved in the generation of ventral midbrain neuronal cell types in vitro and in vivo [38-41]. One of the major functions of Shh in the developing nervous system is to specify ventral cell types of the neural tube $[38,42]$. FGF8 is involved in the patterning of the isthmus, a region that divides the midbrain and hindbrain regions during brain development [43]. Together, these molecules specify cell types arising within the ventral midbrain regions of the embryo and thus are used in vitro to specify neural stem/ progenitor cells to midbrain dopaminergic neurons. The timing of Shh and FGF8 exposure during neural differentiation may be important. Most protocols add Shh and 
FGF8 to culture media during the expansion period of neural progenitors, and/or in the early stages of neuronal differentiation [39-41]. One study showed that if hESCderived neural progenitors are initially exposed to FGF2 treatment and then later followed by FGF8 and Shh, this gave rise to forebrain-like dopaminergic neurons (fig. 2). Whereas early exposure of progenitors to FGF8 rather than FGF2, followed by FGF8 and Shh, generated midbrain dopaminergic neurons [39]. As mentioned above, Elkabetz et al. [27] described how Shh and Notch signalling helped maintain R-NSCs undifferentiated. In this study, they found that long-term addition of FGF8 to RNSC cultures promoted their differentiation. Thus, the functional outcome of Shh and FGF8 signalling may be dependent upon the stage of neural differentiation. Overall, the proportion of dopaminergic neurons obtained may vary between different protocols and is probably dependent upon the hESC line, hESC culture conditions and their system of neural induction. Nevertheless, these signalling factors that are shown to enhance specification of midbrain dopamine neurons during embryogenesis also seem to influence neuronal fate during hESC neural differentiation.

One issue relating to using exogenous factors as a strategy to generate midbrain dopamine neurons is that these factors also are involved in the generation of other neuronal populations such as serotonin neurons [44]. Thus, the other approach for generating specific cell types is to use intrinsic factors in addition to, or perhaps alternatively, extrinsic factors. As outlined above, during development progenitors of midbrain dopamine neurons are identified by their expression of Pitx 3, Lmxla, Nurr1, En1, and Foxa2 transcription factors during different stages of differentiation. Recent studies found that forced Lmxla expression in hESC-derived neural progenitors all gave rise to midbrain dopamine neurons [45]. This is useful as a 'proof of concept' approach to generate specified neural progenitors in vitro to determine whether they give rise to midbrain dopamine neurons in vivo. Indeed, this was observed using mouse ESC-derived neural progenitors that had forced expression of Lmxla [45]. Identification of cell surface markers that are specific to committed neural progenitor cell types, such as midbrain dopamine progenitor, will be useful for isolating specific cell populations without genetic manipulation.

\section{Spinal Cord Motor Neurons}

hESC neural induction by noggin treatment, stromal co-cultures or embryoid bodies results in neural progenitors characteristic of forebrain/midbrain and dorsal neural tube cell types [11, 20,27]. Therefore, generation of spinal cord motor neurons requires signals to direct the fate of neural progenitors to more caudal and ventral cell types $[8,20]$. A study by $\mathrm{Li}$ et al. [20] described when commitment of progenitor fate occurs following neural induction. During neural induction using a chemically defined media adherent culture system, initially rosettes are Pax6+/Sox1- and then later in culture become Pax6+/ Sox $1+$. Early rosettes also possess a rostral orientation and can be identified by the expression of Otx2, a forebrain/midbrain marker, and are negative for the midbrain marker Engrailed1 and HoxC8, a homeodomain protein produced in the spinal cord [20]. Further differentiation of neural cultures also reveals the upregulation of Pax7, a dorsally expressed transcription factor. This study showed that early rosettes can be caudalized when exposed to RA (fig. 2) [20]. The exposure of Sox1+ late rosettes to RA does not influence their rostral-caudal orientation and suggests that Sox1-expressing rosettes are already regionally specified. Shh was added during the later stages of neural differentiation to induce specification of ventral cell types, in particular motor neurons. Another study published later showed that the small molecule, purmorphamine, functions as a Shh agonist and could be exchanged for Shh in the culture system to generate motor neurons [46].

It is interesting that, in both systems for generating midbrain dopamine neurons and spinal cord motor neurons, the signals responsible for specifying the rostralcaudal fate of progenitors, FGF8 and RA, respectively, need to be added at early stages during neural induction $[20,39]$. Whereas the signal required for ventralizing progenitor fate, Shh, was added at slightly later stages of neural differentiation [20,39]. Indeed, dorsal markers such as Pax7 are observed to be expressed at later stages during stages of neural progenitor expansion [11]. Thus, rostralcaudal and dorsal-ventral patterning signals may also require appropriate timing and co-ordination of exposure for their function in driving progenitor cell fate to be effective.

\section{Conclusions}

Although different protocols have been employed to direct hESC neural differentiation, there are common signals and stages observed across all culture systems. hESC neural differentiation can be crudely divided into three main stages of neural induction, neural stem/progenitor expansion, and neuronal and glial differentia- 
tion. However, as each of these stages is more carefully characterized, it is evident that each stage can be further subdivided into multiple events to progress a stem cell through its different progenitor stages. Identifying the signals and mechanisms involved at each of these stages is important for learning how to ultimately manipulate stem cells, whether it be from an endogenous or exogenous source of cells, to restore and repair neural circuitry.

\section{References}

- 1 Thomson JA, Itskovitz-Eldor J, Shapiro SS, Waknitz MA, Swiergiel JJ, Marshall VS, Jones JM: Embryonic stem cell lines derived from human blastocysts. Science 1998;282: $1145-1147$.

-2 Reubinoff BE, Itsykson P, Turetsky T, Pera MF, Reinhartz E, Itzik A, Ben-Hur T: Neural progenitors from human embryonic stem cells. Nat Biotechnol 2001;19:1134-1140.

-3 Zhang SC, Wernig M, Duncan ID, Brustle O, Thomson JA: In vitro differentiation of transplantable neural precursors from human embryonic stem cells. Nat Biotechnol 2001;19:1129-1133.

-4 Koch P, Opitz T, Steinbeck JA, Ladewig J, Brustle O: A rosette-type, self-renewing human ES cell-derived neural stem cell with potential for in vitro instruction and synaptic integration. Proc Natl Acad Sci USA 2009; 106:3225-3230

5 Carpenter MK, Inokuma MS, Denham J, Mujtaba T, Chiu CP, Rao MS: Enrichment of neurons and neural precursors from human embryonic stem cells. Exp Neurol 2001;172: 383-397.

-6 Schuldiner M, Eiges R, Eden A, Yanuka O, Itskovitz-Eldor J, Goldstein RS, Benvenisty $\mathrm{N}$ : Induced neuronal differentiation of human embryonic stem cells. Brain Res 2001; 913:201-205.

7 Pomp O, Brokhman I, Ben-Dor I, Reubinoff B, Goldstein RS: Generation of peripheral sensory and sympathetic neurons and neural crest cells from human embryonic stem cells. Stem Cells 2005;23:923-930.

-8 Lee G, Kim H, Elkabetz Y, Al Shamy G, Panagiotakos G, Barberi T, Tabar V, Studer L: Isolation and directed differentiation of neural crest stem cells derived from human embryonic stem cells. Nat Biotechnol 2007;25: 1468-1475.

\9 Chambers SM, Fasano CA, Papapetrou EP, Tomishima M, Sadelain M, Studer L: Highly efficient neural conversion of human ES and iPS cells by dual inhibition of SMAD signaling. Nat Biotechnol 2009;27:275-280.

$\checkmark 10$ Pera MF, Andrade J, Houssami S, Reubinoff B, Trounson A, Stanley EG, Ward-van Oostwaard D, Mummery C: Regulation of human embryonic stem cell differentiation by BMP2 and its antagonist noggin. J Cell Sci 2004; 117:1269-1280.
1 Davidson KC, Jamshidi P, Daly R, Hearn MT, Pera MF, Dottori M: Wnt3a regulates survival, expansion, and maintenance of neural progenitors derived from human embryonic stem cells. Mol Cell Neurosci 2007; 36:408-415.

12 Dottori M, Pera MF: Neural differentiation of human embryonic stem cells. Methods Mol Biol 2008;438:19-30.

13 Yao S, Chen S, Clark J, Hao E, Beattie GM, Hayek A, Ding S: Long-term self-renewal and directed differentiation of human embryonic stem cells in chemically defined conditions. Proc Natl Acad Sci USA 2006; 103:6907-6912.

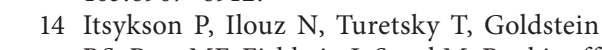
RS, Pera MF, Fishbein I, Segal M, Reubinoff BE: Derivation of neural precursors from human embryonic stem cells in the presence of noggin. Mol Cell Neurosci 2005;30:2436.

-23 Kawasaki H, Mizuseki K, Nishikawa S, Kaneko S, Kuwana Y, Nakanishi S, Nishikawa SI, Sasai Y: Induction of midbrain dopaminergic neurons from ES cells by stromal cell-derived inducing activity. Neuron 2000; 28:31-40.

24 Kitajima H, Yoshimura S, Kokuzawa J, Kato M, Iwama T, Motohashi T, Kunisada T, Sakai $\mathrm{N}$ : Culture method for the induction of neurospheres from mouse embryonic stem cells by coculture with PA6 stromal cells. J Neurosci Res 2005;80:467-474.

25 Reynolds BA, Tetzlaff W, Weiss S: A multipotent EGF-responsive striatal embryonic progenitor cell produces neurons and astrocytes. J Neurosci 1992;12:4565-4574.

26 Reynolds BA, Weiss S: Clonal and population analyses demonstrate that an EGF-responsive mammalian embryonic CNS precursor is a stem cell. Dev Biol 1996;175: 1-13.

15 Wu MY, Hill CS: Tgf-beta superfamily signaling in embryonic development and homeostasis. Dev Cell 2009;16:329-343.

16 Chen D, Zhao M, Mundy GR: Bone morphogenetic proteins. Growth Factors 2004;22: 233-241.

17 Gajović S, St-Onge L, Yokota Y, Gruss P: Retinoic acid mediates Pax6 expression during in vitro differentiation of embryonic stem cells. Differentiation 1997;62:187-192.

18 Okada Y, Shimazaki T, Sobue G, Okano H: Retinoic-acid-concentration-dependent acquisition of neural cell identity during in vitro differentiation of mouse embryonic stem cells. Dev Biol 2004;275:124-142.

19 Kawamorita M, Suzuki C, Saito G, Sato T, Sato K: In vitro differentiation of mouse embryonic stem cells after activation by retinoic acid. Hum Cell 2002;15:178-182.

20 Li XJ, Du ZW, Zarnowska ED, Pankratz M, Hansen LO, Pearce RA, Zhang SC: Specification of motoneurons from human embryonic stem cells. Nat Biotechnol 2005;23:215221.

-21 Okita K, Yamanaka S: Intracellular signaling pathways regulating pluripotency of embryonic stem cells. Curr Stem Cell Res Ther 2006;1:103-111.

22 Gratsch TE, O'Shea KS: Noggin and chordin have distinct activities in promoting lineage commitment of mouse embryonic stem (ES) cells. Dev Biol 2002;245:83-94.
Elkabetz Y, Panagiotakos G, Al Shamy G, Socci ND, Tabar V, Studer L: Human ES cellderived neural rosettes reveal a functionally distinct early neural stem cell stage. Genes Dev 2008;22:152-165.

28 Takuwa Y, Takuwa N, Sugimoto N: The EDG family G protein-coupled receptors for lysophospholipids: their signaling properties and biological activities. J Biochem 2002;131: 767-771.

29 Dottori M, Leung J, Turnley AM, Pebay A: Lysophosphatidic acid inhibits neuronal differentiation of neural stem/progenitor cells derived from human embryonic stem cells. Stem Cells 2008;26:1146-1154.

30 Newman MB, Bakay RA: Therapeutic potentials of human embryonic stem cells in Parkinson's disease. Neurotherapeutics 2008;5: 237-251.

31 Guo C, Qiu HY, Shi M, Huang Y, Johnson RL, Rubinstein M, Chen SD, Ding YQ: Lmxlb-controlled isthmic organizer is essential for development of midbrain dopaminergic neurons. J Neurosci 2008;28: 14097-14106.

32 Smidt MP, Smits SM, Bouwmeester H, Hamers FP, van der Linden AJ, Hellemons AJ, Graw J, Burbach JP: Early developmental failure of substantia nigra dopamine neurons in mice lacking the homeodomain gene Pitx3. Development 2004;131:1145-1155. 
- 33 Jorgensen JR, Juliusson B, Henriksen KF, Hansen C, Knudsen S, Petersen TN, Blom N, Seiger A, Wahlberg LU: Identification of novel genes regulated in the developing human ventral mesencephalon. Exp Neurol 2006;198:427-437.

- 34 Ferri AL, Lin W, Mavromatakis YE, Wang JC, Sasaki H, Whitsett JA, Ang SL: Foxa1 and Foxa2 regulate multiple phases of midbrain dopaminergic neuron development in a dosage-dependent manner. Development 2007; 134:2761-2769.

-35 Ang SL: Transcriptional control of midbrain dopaminergic neuron development. Development 2006;133:3499-3506.

- 36 Simon HH, Saueressig H, Wurst W, Goulding MD, O'Leary DD: Fate of midbrain dopaminergic neurons controlled by the engrailed genes. J Neurosci 2001;21:31263134.

37 Smits SM, Burbach JP, Smidt MP: Developmental origin and fate of meso-diencephalic dopamine neurons. Prog Neurobiol 2006;78: $1-16$.
38 Roussa E, Krieglstein K: Induction and specification of midbrain dopaminergic cells: focus on Shh, FGF8, and TGF-beta. Cell Tissue Res 2004;318:23-33.

39 Yan Y, Yang D, Zarnowska ED, Du Z, Werbel B, Valliere C, Pearce RA, Thomson JA, Zhang SC: Directed differentiation of dopaminergic neuronal subtypes from human embryonic stem cells. Stem Cells 2005;23: 781-790.

40 Perrier AL, Tabar V, Barberi T, Rubio ME, Bruses J, Topf N, Harrison NL, Studer L: Derivation of midbrain dopamine neurons from human embryonic stem cells. Proc Natl Acad Sci USA 2004;101:12543-12548.

41 Cho MS, Hwang DY, Kim DW: Efficient derivation of functional dopaminergic neurons from human embryonic stem cells on a large scale. Nat Protoc 2008;3:1888-1894.

42 Chamberlain CE, Jeong J, Guo C, Allen BL, McMahon AP: Notochord-derived Shh concentrates in close association with the apically positioned basal body in neural target cells and forms a dynamic gradient during neural patterning. Development 2008;135: 1097-1106.
43 Nakamura H, Sato T, Suzuki-Hirano A: Isthmus organizer for mesencephalon and metencephalon. Dev Growth Differ 2008; 50(suppl 1):S113-S118.

44 Ye W, Shimamura K, Rubenstein JL, Hynes MA, Rosenthal A: FGF and Shh signals control dopaminergic and serotonergic cell fate in the anterior neural plate. Cell 1998;93: 755-766.

45 Friling S, Andersson E, Thompson LH, Jonsson ME, Hebsgaard JB, Nanou E, Alekseenko Z, Marklund U, Kjellander S, Volakakis N, Hovatta O, El Manira A, Bjorklund A, Perlmann T, Ericson J: Efficient production of mesencephalic dopamine neurons by Lmxla expression in embryonic stem cells. Proc Natl Acad Sci USA 2009;106:76137618.

46 Li XJ, Hu BY, Jones SA, Zhang YS, Lavaute T, Du ZW, Zhang SC: Directed differentiation of ventral spinal progenitors and motor neurons from human embryonic stem cells by small molecules. Stem Cells 2008;26:886893. 